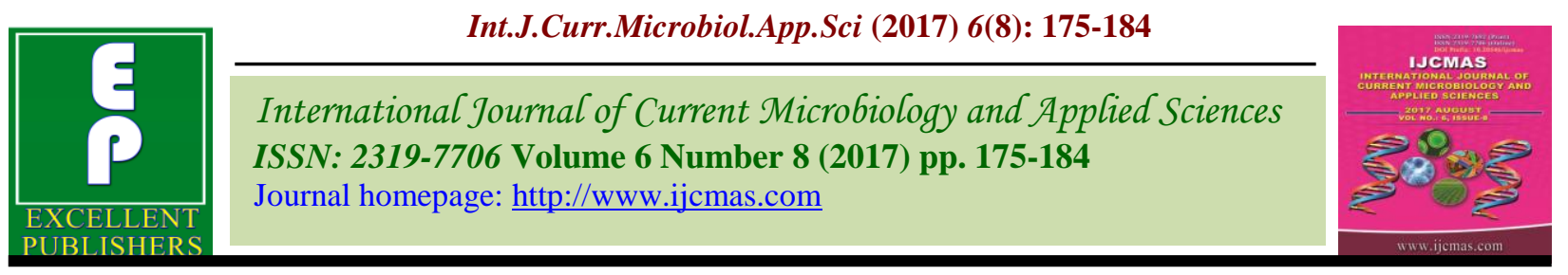

Original Research Article

https://doi.org/10.20546/ijcmas.2017.608.025

\title{
Character Association and Path Analysis for Quantitative Traits in Garlic (Allium sativum L.)
}

\author{
Pooja Chotaliya* and G.U. Kulkarni
}

\author{
Department of Genetics and Plant Breeding, J.A.U., Junagadh, Gujarat, India \\ *Corresponding author
}

\section{A B S T R A C T}

\begin{tabular}{|l|}
\hline Ke y w or d s \\
Garlic, Traits, \\
Correlation, \\
Path analysis. \\
\hline Article Info \\
\hline Accepted: \\
04 June 2017 \\
Available Online: \\
10 August 2017 \\
\hline
\end{tabular}

An experiment was conducted to assess correlation and path analysis in 156 genotypes and 4 checks of garlic grown in Augmented Randomized Block Design at Vegetable Research Station, Junagadh Agricultural University, Junagadh during rabi season of 2015-2016. The observations were recorded on 18 characters viz., plant height $(\mathrm{cm})$, number of leaves per plant, leaf length $(\mathrm{cm})$, leaf width at middle portion $(\mathrm{cm})$, days to maturity (days), pseudostem height $(\mathrm{cm})$, collar thickness $(\mathrm{cm})$, bulb collar diameter $(\mathrm{cm})$, bulb equatorial diameter $(\mathrm{cm})$, bulb polar diameter $(\mathrm{cm})$, bulb weight $(\mathrm{g})$, number of cloves per bulb, clove weight $(\mathrm{g})$, clove length $(\mathrm{cm})$, clove polar diameter $(\mathrm{cm})$, clove equatorial diameter $(\mathrm{cm})$, total soluble solids (\%) and bulb yield ( $\mathrm{kg} / \mathrm{ha})$. In general, the estimates of genotypic correlation were higher than the corresponding phenotypic correlation coefficient. It may result from the modifying effect of environment on the association of characters at genotypic level. The bulb yield had significant positive correlation with characters like with plant height, number of leaves per plant, leaf width at middle portion, pseudostem length, bulb collar diameter, bulb equatorial diameter, bulb polar diameter, bulb weight and clove length, clove polar diameter and clove equatorial diameter. From these associations, it appears that higher bulb yield can be obtained by increasing these characters. Path coefficient analysis revealed that the characters like plant height and clove polar diameter had high direct effect on bulb yield. These above characters also had positive indirect effect on each other. Plant height, number of leaves per plant, leaf width at middle portion, pseudostem height exhibited positive and indirect effects on bulb yield via plant height, leaf length, leaf width at middle portion, days to maturity, pseudostem height, bulb equatorial diameter, bulb polar diameter, bulb weight, number of cloves per bulb, clove weight and clove polar diameter, clove equatorial diameter and total soluble solids. Bulb collar diameter, bulb equatorial diameter, bulb polar diameter, bulb weight, clove length, clove polar diameter and clove equatorial diameter contributed indirectly by giving positive indirect effects on bulb yield through plant height, leaf width at middle portion, bulb equatorial diameter, bulb polar diameter, bulb weight, number of cloves per bulb, clove polar diameter, clove equatorial diameter and total soluble solids.

\section{Introduction}

Garlic (Allium sativum L.) is one of the most important remunerative bulbous spice and medicinal crop grown commercially. It is the seconf most widely used spice after onion. It belongs to family Alliaceae because of its

superior ovary and scapose umbeliate inflorescence. The name garlic is derived from the old English word "gar" meaning spear presumably referring to clove. Allium longicupis is considered to be its wild 
ancestor, which is extensively found in Central Asia. Garlic (Allium sativum L.) has $2 n=2 x=16$ chromosome number. Garlic is a perennial plant, cultivated mainly for bulbs. The edible underground stem is the composite bulb made up of numerous smaller bulbs called clove. Leaves have solid thin blades. Some varieties produce flowers but these flowers do not set seeds. There are some varieties which produce vegetative bulbs on the top called bulbils. They are also used for propagation in some cases.

The basic rationale in any crop improvement programme is the increase in yield potential of the crop. The character bulb yield has got a complex gene action and so, in order to study it properly, different factors affecting the yield must be considered and evaluated with regard to their contribution to yield. Knowledge in respect of nature and magnitude of association with different component characters is a pre-requisite to bring the improvement in desired direction. In order to accumulate optimum combination of bulb yield contributing characters in a single genotype, it is essential to know the relationships among themselves.

Further, the bulb yield is influenced by its various components directly and or indirectly via other traits that create a complex situation before a breeder for making selection. Therefore, path coefficient analysis could provide a more realistic picture of the inter relationship, as it partitions the correlation in direct and indirect effect of the variables.

\section{Materials and Methods}

One hundred and fifty six genotypes and four checks of garlic were sown in an Augmented Randomized Block Design (ARBD) (Federer, 1956) during rabi season of 2015-2016. The cloves were planted at a distance of $15 \mathrm{~cm}$ row to row and $10 \mathrm{~cm}$ plant to plant as per spacing. Other recommended agronomical practices and plant protection measures were followed for the successful raising of the crop. The observations were recorded on various quantitative characters viz., plant height $(\mathrm{cm})$, number of leaves per plant, leaf length $(\mathrm{cm})$, leaf width at middle portion (cm), days to maturity (days), pseudostem height $(\mathrm{cm})$, collar thickness $(\mathrm{cm})$, bulb collar diameter $(\mathrm{cm})$, bulb equatorial diameter $(\mathrm{cm})$, bulb polar diameter $(\mathrm{cm})$, bulb weight $(\mathrm{g})$, number of cloves per bulb, clove weight $(\mathrm{g})$, clove length $(\mathrm{cm})$, clove polar diameter $(\mathrm{cm})$, clove equatorial diameter $(\mathrm{cm})$, total soluble solids (\%) and bulb yield (kg/ha). were recorded on five competitive plants per genotype in each plot were randomly selected for the purpose of recording observations on different characters (except days to maturity) and their averages were used in the statistical analysis.

Days to maturity was measured on plot basis. The phenotypic and genotypic correlation coefficients of all the characters were workedout as per Al-Jibouri et al., (1958). The path coefficient analysis was carried out as per the method suggested by Dewey and Lu (1959). Phenotypic correlation coefficients of 17 variables with bulb yield were used to estimate the path coefficients for the direct effects of various independent characters on bulb yield.

\section{Results and Discussion}

The correlation coefficients were worked out among 18 characters to find out association of bulb yield with its components at genotypic $\left(r_{g}\right)$ and phenotypic $\left(r_{p}\right)$ levels. The data given in (Table 1) revealed that in general, the genotypic correlation coefficients were higher than the respective phenotypic correlations which might be from modifying effect of environment on the association of characters at genotypic level. Selection of yield as such 
may not be effective since there may be number of genes for bulb yield and bulb yield may be resultant of interaction among its various components. Knowledge of relation between bulb yield and its component is essential and selection for one component may bring about a simultaneous change in the other.

Therefore, for a rational approach to improve bulb yield, it may be useful to collect information on character association. Results of genotypic and phenotypic correlation analysis of present study are graphically represented in figures 1 and 2.

The correlation of bulb yield showed positive and significant at genotypic and phenotypic level with plant height, number of leaves per plant, leaf width at middle portion, pseudostem length, bulb collar diameter, bulb equatorial diameter, bulb polar diameter, bulb weight and clove length, clove polar diameter and clove equatorial diameter, indicating that selection based on these traits will help increasing the yield of garlic.

Similar result was found by Moravec et al., (1974), Singh (1981), Kalloo et al., (1982), Singh (1984), Rahman and Das (1985), Lokhande and Pawar (1988), Kohli and Fageria (1992), Kohli and Mahajan (1993), Srivastava et al., (1993), Baiday and Tiwari (1995), Thakur et al., (1997), Agrawal (1999), Khan (2002), Agrawal and Tiwari (2009), Tsega et al., (2010), Singh et al., (2012), Sonkiya et al., (2012), Idress Al Gehani et al., (2013), Panse et al., (2013), Singh et al., (2013), Pervin et al., (2014), Samaptika Kar et al., (2014), Kadam et al., (2016), Prajapati et al., (2016), Bhatt et al., (2017) in garlic.

Positive association of bulb weight was found positive and significant at genotypic and phenotypic level with plant height, number of leaves per plant, leaf width at middle portion, pseudostem length, collar thickness, bulb collar diameter, bulb equatorial diameter, bulb polar diameter. Similar findings were reported by Moravec et al., (1974), Singh (1981), Shaha et al., (1990), Kohli and Mahajan (1993), Srivastava et al., (1993), Barman et al., (1996), Dhall et al., (2013), Esho (2015), Satesh Kumar et al., (2015) and Sharma et al., (2016) in garlic.

The correlation of plant height showed positive and significant at genotypic and phenotypic level with leaf length, collar thickness, bulb weight, number of cloves per bulb, bulb yield indicating that selection based on these traits will help increasing the plant height of garlic.

Similar result was found by Singh (1981), Shaha et al., (1990), Kohli and Fageria (1992), Kohli and Mahajan (1993), Barman et al., (1996) and Esho (2015).

Generally, the value of correlation coefficients lies between -1.0 to 1.0 . In the present study all the values of correlation coefficient ranged from -1.0 to 1.0 except one case, the value of correlation coefficient was more than 1; it may be due to when covariance is over estimated whereas variance is under estimated (Roy, 2000).

When two or more variables are included in the correlation studies, it becomes difficult to determine which characters enhance the yield. The technique of path coefficient analysis overcomes this situation which partitions the forces of association and examines the relative contribution of direct and indirect effects of the independent variables on the dependent variables. The direct and indirect effects of variance characters along with bulb yield are executed in table 2 .

The characters, which had shown significant phenotypic correlation with bulb yield, were considered for the results as well as discussion. 
Table.1 Phenotypic (P) and genotypic (G) correlation coefficients between different characters in garlic

\begin{tabular}{|c|c|c|c|c|c|c|c|c|c|c|c|c|c|c|c|c|c|c|c|}
\hline Characters & & 1 & 2 & 3 & 4 & 5 & 6 & 7 & 8 & 9 & 10 & 11 & 12 & 13 & 14 & 15 & 16 & 17 & 18 \\
\hline \multirow[t]{2}{*}{1} & $\mathbf{P}$ & 1.000 & 0.145 & $0.643 * *$ & $0.440 * *$ & 0.005 & 0.159 & $0.590 * *$ & $0.268 * *$ & $0.275 * *$ & 0.153 & $0.411 * *$ & $0.512 * *$ & -0.033 & 0.065 & 0.052 & -0.102 & -0.005 & $0.268 * *$ \\
\hline & $\mathbf{G}$ & 1.000 & $-0.496^{* *}$ & $0.275^{* *}$ & 0.113 & $-0.629 * *$ & $0.312 * *$ & $0.395 * *$ & 0.093 & -0.061 & -0.013 & $0.904 * *$ & $2.076 * *$ & -0.065 & $1.162 * *$ & $0.873 * *$ & $-0.223 * *$ & -0.027 & $0.702 * *$ \\
\hline \multirow[t]{2}{*}{2} & $\mathbf{P}$ & & 1.000 & -0.066 & $0.369 * *$ & 0.091 & 0.021 & 0.045 & 0.109 & $0.378 * *$ & $0.217 * *$ & $0.386 * *$ & 0.097 & 0.137 & $0.416 * *$ & $0.455^{* *}$ & $0.365 * *$ & 0.099 & $0.209 * *$ \\
\hline & $\mathbf{G}$ & & 1.000 & $-0.734 * *$ & $0.449 * *$ & $0.486^{* *}$ & $0.176^{*}$ & $-0.207 *$ & $-0.177^{*}$ & $0.421 * *$ & $0.312 * *$ & $0.619 * *$ & $0.395 * *$ & $0.258 * *$ & $1.185 * *$ & $1.151 * *$ & $0.613 * *$ & 0.124 & $0.345 * *$ \\
\hline \multirow[t]{2}{*}{3} & $\mathbf{P}$ & & & 1.000 & $0.392 * *$ & -0.060 & $-0.329 * *$ & $0.499 * *$ & $0.188^{*}$ & 0.036 & -0.057 & 0.134 & $0.430 * *$ & $-0.197 *$ & -0.139 & -0.136 & $-0.235 * *$ & -0.059 & 0.042 \\
\hline & $\mathbf{G}$ & & & 1.000 & $0.410 * *$ & $-0.626 * *$ & $-0.618 * *$ & $0.456 * *$ & $0.235^{* *}$ & $-0.178 *$ & $-0.195^{*}$ & $0.237 * *$ & $1.444 * *$ & $-0.335 * *$ & $0.210 * *$ & $0.199 *$ & $-0.390 * *$ & -0.098 & 0.051 \\
\hline \multirow[t]{2}{*}{4} & $\mathbf{P}$ & & & & 1.000 & 0.153 & $-0.012 * *$ & $0.321 * *$ & $0.441 * *$ & $0.417 * *$ & $0.359 * *$ & $0.323 * *$ & 0.147 & 0.031 & $0.330 * *$ & $0.266 * *$ & $0.223^{* *}$ & -0.024 & $0.245 * *$ \\
\hline & $\mathbf{G}$ & & & & 1.000 & -0.079 & $-0.145 * *$ & $0.228 * *$ & $0.878 * *$ & $0.584 * *$ & $0.538 * *$ & $0.482 * *$ & $0.465 * *$ & 0.083 & $0.920 * *$ & $0.643 * *$ & $0.345 * *$ & -0.053 & $0.379 * *$ \\
\hline \multirow[t]{2}{*}{5} & $\mathbf{P}$ & & & & & 1.000 & -0.005 & 0.039 & 0.086 & 0.082 & 0.107 & 0.011 & -0.048 & -0.050 & 0.094 & 0.095 & 0.053 & 0.051 & 0.020 \\
\hline & $\mathbf{G}$ & & & & & 1.000 & 0.127 & 0.004 & $0.905 * *$ & $1.575 * *$ & $1.068 * *$ & $-0.310 * *$ & $-1.314 * *$ & -0.123 & $0.935 * *$ & $0.830 * *$ & $0.485 * *$ & $0.363 * *$ & $0.631 * *$ \\
\hline \multirow[t]{2}{*}{6} & $\mathbf{P}$ & & & & & & 1.000 & 0.065 & -0.039 & 0.097 & $0.168 *$ & $0.166^{*}$ & -0.061 & $0.184 *$ & 0.139 & 0.106 & 0.118 & -0.088 & $0.185^{*}$ \\
\hline & $\mathbf{G}$ & & & & & & 1.000 & 0.046 & -0.077 & $0.175^{*}$ & $0.223 * *$ & $0.240 * *$ & $-0.218 * *$ & 0.153 & $0.114 *$ & 0.013 & 0.040 & -0.107 & 0.129 \\
\hline \multirow[t]{2}{*}{7} & $\mathbf{P}$ & & & & & & & 1.000 & $0.308 * *$ & $0.236^{* *}$ & 0.077 & $0.289 * *$ & $0.343 * *$ & 0.012 & 0.021 & 0.018 & -0.050 & -0.075 & 0.019 \\
\hline & $\mathbf{G}$ & & & & & & & 1.000 & 0.152 & 0.046 & $-0.286^{* *}$ & $0.543 * *$ & $1.082 * *$ & -0.021 & $0.480 * *$ & $0.232 * *$ & $-0.168 *$ & -0.157 & 0.045 \\
\hline \multirow[t]{2}{*}{8} & $\mathbf{P}$ & & & & & & & & 1.000 & $0.535^{* *} *$ & $0.535^{* *}$ & $0.354 * *$ & 0.122 & 0.142 & $0.354 * *$ & $0.368 * *$ & $0.268 * *$ & $0.2130 *$ & $0.160 *$ \\
\hline & $\mathbf{G}$ & & & & & & & & 1.000 & $0.566 * *$ & $0.832 * *$ & $0.671 * *$ & 0.010 & $0.281 * *$ & $0.911 * *$ & $0.849 * *$ & $0.629 * *$ & $0.325 * *$ & $0.209 * *$ \\
\hline \multirow[t]{2}{*}{9} & $\mathbf{P}$ & & & & & & & & & 1.000 & $0.734 * *$ & $0.656^{* *}$ & 0.147 & $0.318 * *$ & $0.588 * *$ & $0.611 * *$ & $0.487 * *$ & 0.092 & $0.304 * *$ \\
\hline & $\mathbf{G}$ & & & & & & & & & 1.000 & $0.802 * *$ & $1.314 * *$ & $0.664 * *$ & $0.500 * *$ & $1.699 * *$ & $1.579 * *$ & $0.874 * *$ & 0.135 & $0.461 * *$ \\
\hline 10 & $\mathbf{P}$ & & & & & & & & & & 1.000 & $0.350 * *$ & -0.072 & $0.234 * *$ & $0.519 * *$ & $0.516 * *$ & $0.474 * *$ & 0.010 & $0.257 * *$ \\
\hline \multirow[t]{2}{*}{11} & $\mathbf{P}$ & & & & & & & & & & & 1.000 & $0.517 * *$ & $0.266^{* *}$ & $0.432 * *$ & $0.461 * *$ & $0.232 * *$ & 0.133 & $0.300 * *$ \\
\hline & $\mathbf{G}$ & & & & & & & & & & & 1.000 & $0.654 * *$ & $0.270 * *$ & $0.715^{* *}$ & $0.637 * *$ & $0.204 *$ & $0.175^{*}$ & $0.400 * *$ \\
\hline \multirow[t]{2}{*}{12} & $\mathbf{P}$ & & & & & & & & & & & & 1.000 & $-0.167 *$ & 0.016 & -0.010 & $-0.278 * *$ & 0.132 & 0.112 \\
\hline & $\mathbf{G}$ & & & & & & & & & & & & 1.000 & $-0.335 * *$ & $-0.254 * *$ & $-0.341 * *$ & $-0.514 * *$ & 0.158 & -0.122 \\
\hline \multirow[t]{2}{*}{13} & $\mathbf{P}$ & & & & & & & & & & & & & 1.000 & $0.225^{* *}$ & $0.264 * *$ & $0.431 * *$ & 0.088 & 0.078 \\
\hline & $\mathbf{G}$ & & & & & & & & & & & & & 1.000 & $0.167 *$ & $0.223 * *$ & $0.404 * *$ & 0.097 & 0.056 \\
\hline \multirow[t]{2}{*}{14} & $\mathbf{P}$ & & & & & & & & & & & & & & 1.000 & $0.902 * *$ & $0.546 * *$ & 0.084 & $0.280 * *$ \\
\hline & $\mathbf{G}$ & & & & & & & & & & & & & & 1.000 & $0.898 * *$ & $0.810 * *$ & 0.101 & $0.287 * *$ \\
\hline \multirow[t]{2}{*}{15} & $\mathbf{P}$ & & & & & & & & & & & & & & & 1.000 & $0.579 * *$ & 0.101 & $0.328 * *$ \\
\hline & $\mathbf{G}$ & & & & & & & & & & & & & & & 1.000 & $0.763 * *$ & 0.114 & $0.332 * *$ \\
\hline \multirow[t]{2}{*}{16} & $\mathbf{P}$ & & & & & & & & & & & & & & & & 1.000 & $0.165^{*}$ & $0.225^{* *}$ \\
\hline & $\mathbf{G}$ & & & & & & & & & & & & & & & & 1.000 & $0.213 * *$ & $0.224 * *$ \\
\hline \multirow[t]{2}{*}{17} & $\mathbf{P}$ & & & & & & & & & & & & & & & & & 1.000 & 0.121 \\
\hline & $\mathbf{G}$ & & & & & & & & & & & & & & & & & 1.000 & 0.135 \\
\hline \multirow[t]{2}{*}{18} & $\mathbf{P}$ & & & & & & & & & & & & & & & & & & 1.000 \\
\hline & $\mathbf{G}$ & & & & & & & & & & & & & & & & & & 1.000 \\
\hline
\end{tabular}

*Significant at $\mathrm{p}=0.05$ or at $5 \%$ and $* *$ Significant at $\mathrm{p}=0.01$ or at $1 \%$ (1) Plant height (cm) (2) No. of leaves per plant (3) Leaf length (cm) (4) Leaf width at middle portion $(\mathrm{cm})(5)$ Days to maturity (6) Pseudostem height $(\mathrm{cm})(7)$ Collar thickness $(\mathrm{cm})(8)$ Bulb collar diameter $(\mathrm{cm})(9)$ Bulb equatorial diameter $(\mathrm{cm})$ (10) Bulb polar diameter (cm) (11) Bulb weight (g) (12) No. of cloves per bulb (13) Clove weight (g) (14) Clove length (cm) (15) Clove polar diameter (cm) (16) Clove equatorial diameter (cm) (17) Total Soluble Solids (\%) (18) Bulb yield (kg/ha) 
Table.2 Phenotypic path coefficient analysis showing direct (diagonal and bold) and indirect effects of different Characters on bulb yield of garlic

\begin{tabular}{|c|c|c|c|c|c|c|c|c|c|c|c|c|c|c|c|c|c|c|}
\hline Characters & 1 & 2 & 3 & 4 & 5 & 6 & 7 & 8 & 9 & 10 & 11 & 12 & 13 & 14 & 15 & 16 & 17 & 18 \\
\hline 1 & 0.3108 & -0.0068 & -0.0321 & 0.0656 & -0.001 & 0.0180 & -0.1151 & -0.0197 & 0.0129 & 0.0051 & 0.0284 & 0.0028 & 0.0022 & -0.0161 & 0.0214 & -0.0082 & -0.0005 & $0.2685 * *$ \\
\hline 2 & 0.0452 & -0.0464 & 0.0033 & 0.0550 & -0.0023 & 0.0024 & -0.0088 & -0.0080 & 0.0177 & 0.0073 & 0.0267 & 0.0005 & -0.0091 & -0.1028 & 0.1892 & 0.0292 & 0.0097 & $0.2090 * *$ \\
\hline 3 & 0.2000 & 0.0031 & -0.0499 & 0.0585 & 0.0015 & -0.0370 & -0.0973 & -0.0139 & 0.0017 & -0.0019 & 0.0092 & 0.0024 & 0.0130 & 0.0345 & -0.0568 & -0.0188 & -0.0058 & 0.0426 \\
\hline 4 & 0.1369 & -0.0171 & -0.0196 & 0.1489 & -0.0039 & -0.0013 & -0.0625 & -0.0324 & 0.0196 & 0.0120 & 0.0223 & 0.0008 & -0.0021 & -0.0816 & 0.1105 & 0.0179 & -0.0024 & $0.2459 * *$ \\
\hline 5 & 0.0017 & -0.0042 & 0.0030 & 0.0228 & -0.0253 & -0.0005 & -0.0078 & -0.0064 & 0.0039 & 0.0036 & 0.0008 & -0.0003 & 0.0034 & -0.0231 & 0.0396 & 0.0043 & 0.0050 & 0.0204 \\
\hline 6 & 0.0497 & -0.0010 & 0.0164 & -0.0017 & 0.0001 & 0.1123 & -0.0128 & 0.0029 & 0.0045 & 0.0056 & 0.0115 & -0.0003 & -0.0122 & -0.0344 & 0.0443 & 0.0095 & -0.0086 & $0.1858^{*}$ \\
\hline 7 & 0.1835 & -0.0021 & -0.0249 & 0.0478 & -0.0010 & 0.0074 & -0.1950 & -0.0226 & 0.0111 & 0.0026 & 0.0200 & 0.0019 & -0.0008 & -0.0053 & 0.0083 & -0.0041 & -0.0074 & 0.0195 \\
\hline 8 & 0.0835 & -0.0051 & -0.0094 & 0.0658 & -0.0022 & -0.0044 & -0.0601 & -0.0733 & 0.0251 & 0.0179 & 0.0245 & 0.0007 & -0.0094 & -0.0876 & 0.1528 & 0.0215 & 0.0208 & $0.1609 *$ \\
\hline 9 & 0.0854 & -0.0175 & -0.0018 & 0.0622 & -0.0021 & 0.0109 & -0.0462 & -0.0393 & 0.0468 & 0.0246 & 0.0454 & 0.0008 & -0.0211 & -0.1453 & 0.2538 & 0.0390 & 0.0090 & $0.3047 * *$ \\
\hline 10 & 0.0478 & -0.0101 & 0.0029 & 0.0536 & -0.0027 & 0.0190 & -0.0152 & -0.0392 & 0.0344 & 0.0334 & 0.0243 & -0.0004 & -0.0155 & -0.1283 & 0.2147 & 0.0379 & 0.0010 & $0.2575 * *$ \\
\hline 11 & 0.1276 & -0.0179 & -0.0067 & 0.0480 & -0.0003 & 0.0187 & -0.0565 & -0.0260 & 0.0307 & 0.0117 & 0.0692 & 0.0029 & -0.0177 & -0.1067 & 0.1918 & 0.0186 & 0.0131 & $0.3006^{* *}$ \\
\hline 12 & 0.1592 & -0.0045 & -0.0215 & 0.0219 & 0.0012 & -0.0069 & -0.0669 & -0.0090 & 0.0069 & -0.0024 & 0.0358 & 0.0056 & 0.0111 & -0.0041 & -0.0042 & -0.0223 & 0.0129 & 0.1127 \\
\hline 13 & -0.0102 & -0.0064 & 0.0098 & 0.0047 & 0.0013 & 0.0207 & -0.0022 & -0.0104 & 0.0149 & 0.0078 & 0.0185 & -0.0009 & -0.0661 & -0.0557 & 0.1097 & 0.0344 & 0.0086 & 0.0785 \\
\hline 14 & 0.0202 & -0.0193 & 0.0070 & 0.0492 & -0.0024 & 0.0157 & -0.0042 & -0.0260 & 0.0275 & 0.0174 & 0.0299 & 0.0001 & -0.0149 & -0.2469 & 0.3750 & 0.0437 & 0.0083 & $0.2801 * *$ \\
\hline 15 & 0.0160 & -0.0211 & 0.0068 & 0.0396 & -0.0024 & 0.0120 & -0.0039 & -0.0270 & 0.0286 & 0.0173 & 0.0319 & -0.0001 & -0.0175 & -0.2229 & 0.4153 & 0.0463 & 0.0099 & $0.3289 * *$ \\
\hline 16 & -0.0318 & -0.0170 & 0.0118 & 0.0333 & -0.0013 & 0.0133 & 0.0099 & -0.0197 & 0.0228 & 0.0159 & 0.0161 & -0.0015 & -0.0285 & -0.1350 & 0.2408 & 0.0799 & 0.0161 & $0.2251 * *$ \\
\hline 17 & -0.0017 & -0.0046 & 0.0030 & -0.0036 & -0.0013 & -0.0100 & 0.0148 & -0.0156 & 0.0043 & 0.0004 & 0.0093 & 0.0007 & -0.0059 & -0.0210 & 0.0420 & 0.0132 & 0.0975 & 0.1214 \\
\hline
\end{tabular}

$*$ Significant at $\mathrm{p}=0.05$ or at $5 \%$ and $* *$ Significant at $\mathrm{p}=0.01$ or at $1 \%$

Residual effect: Phenotypic $=0.8664$

N.B.: Values at diagonal and bold indicated direct effects of respective character.

(1) Plant height (cm) (2) No. of leaves per plant (3) Leaf length (cm) (4) Leaf width at middle portion (cm) (5) Days to maturity (6) Pseudostem height (cm) (7) Collar thickness $(\mathrm{cm})(8)$ Bulb collar diameter $(\mathrm{cm})(9)$ Bulb equatorial diameter $(\mathrm{cm})(10)$ Bulb polar diameter (cm) (11) Bulb weight $(\mathrm{g})(12)$ No. of cloves per bulb (13) Clove weight (g) (14) Clove length (cm) (15) Clove polar diameter (cm) (16) Clove equatorial diameter (cm) (17) Total Soluble Solids (\%) (18) Bulb yield $(\mathrm{kg} / \mathrm{ha})$ 
Fig.1 Genotypic correlation coefficient of seventeen component characters with bulb yield

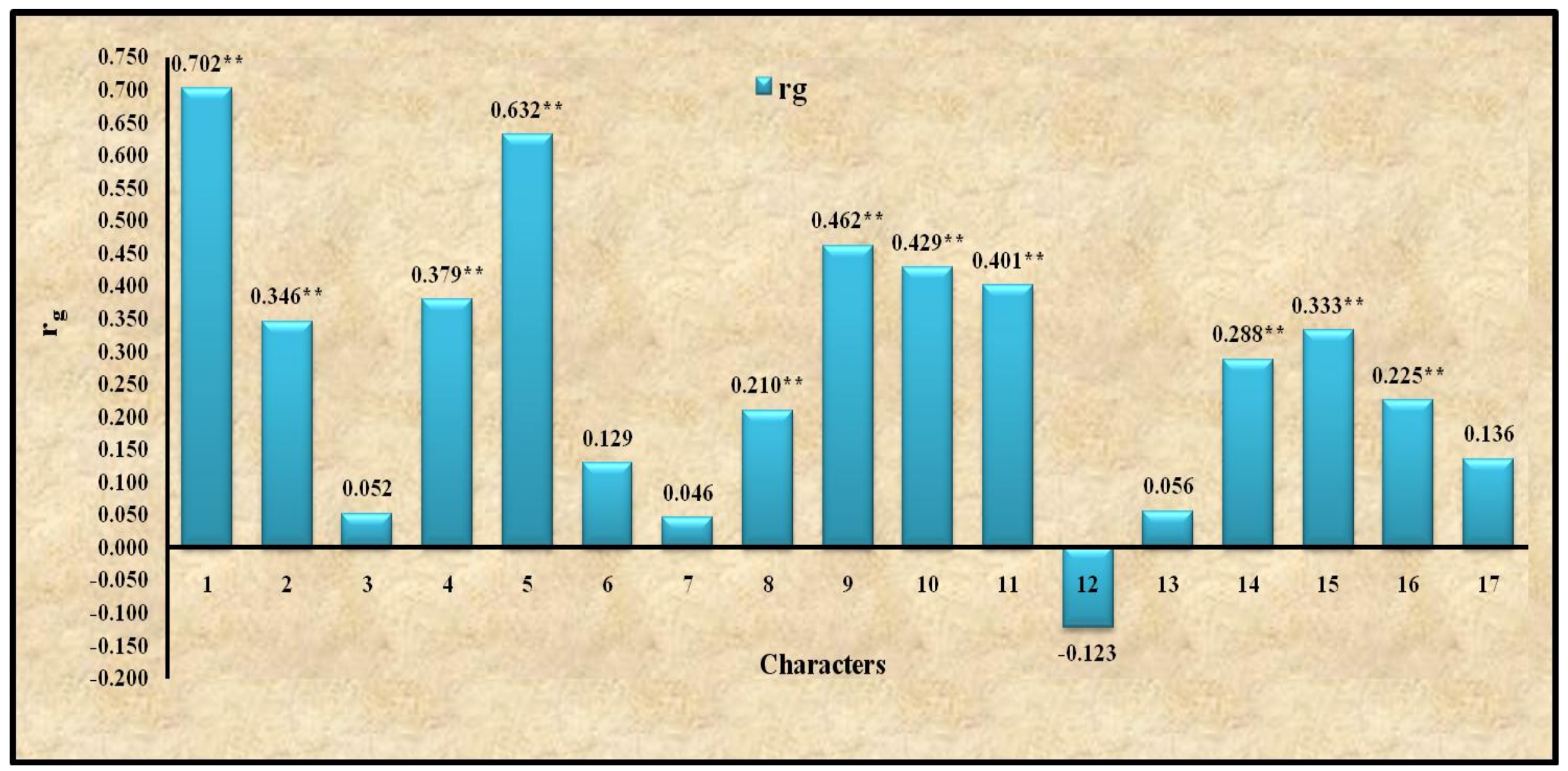

\begin{tabular}{|c|c|c|c|c|c|}
\hline 1. & Plant height $(\mathrm{cm})$ & 2. & No. of leaves per plant & 3. & Leaf length $(\mathrm{cm})$ \\
\hline 4. & Leaf width at middle portion $(\mathrm{cm})$ & 5. & Days to maturity & 6. & Pseudostem height $(\mathrm{cm})$ \\
\hline 7. & Collar thickness $(\mathrm{cm})$ & 8. & Bulb collar diameter $(\mathrm{cm})$ & 9. & Bulb equatorial diameter $(\mathrm{cm})$ \\
\hline 10. & Bulb polar diameter $(\mathbf{c m})$ & 11. & Bulb weight (g) & 12. & No. of cloves per bulb \\
\hline 13. & Clove weight (g) & 14. & Clove length (cm) & 15. & Clove polar diameter $(\mathrm{cm})$ \\
\hline 16. & Clove equatorial diameter & 17. & Total soluble solids $(\%)$ & & \\
\hline
\end{tabular}


Fig.2 Phenotypic correlation coefficient of seventeen component characters with bulb yield

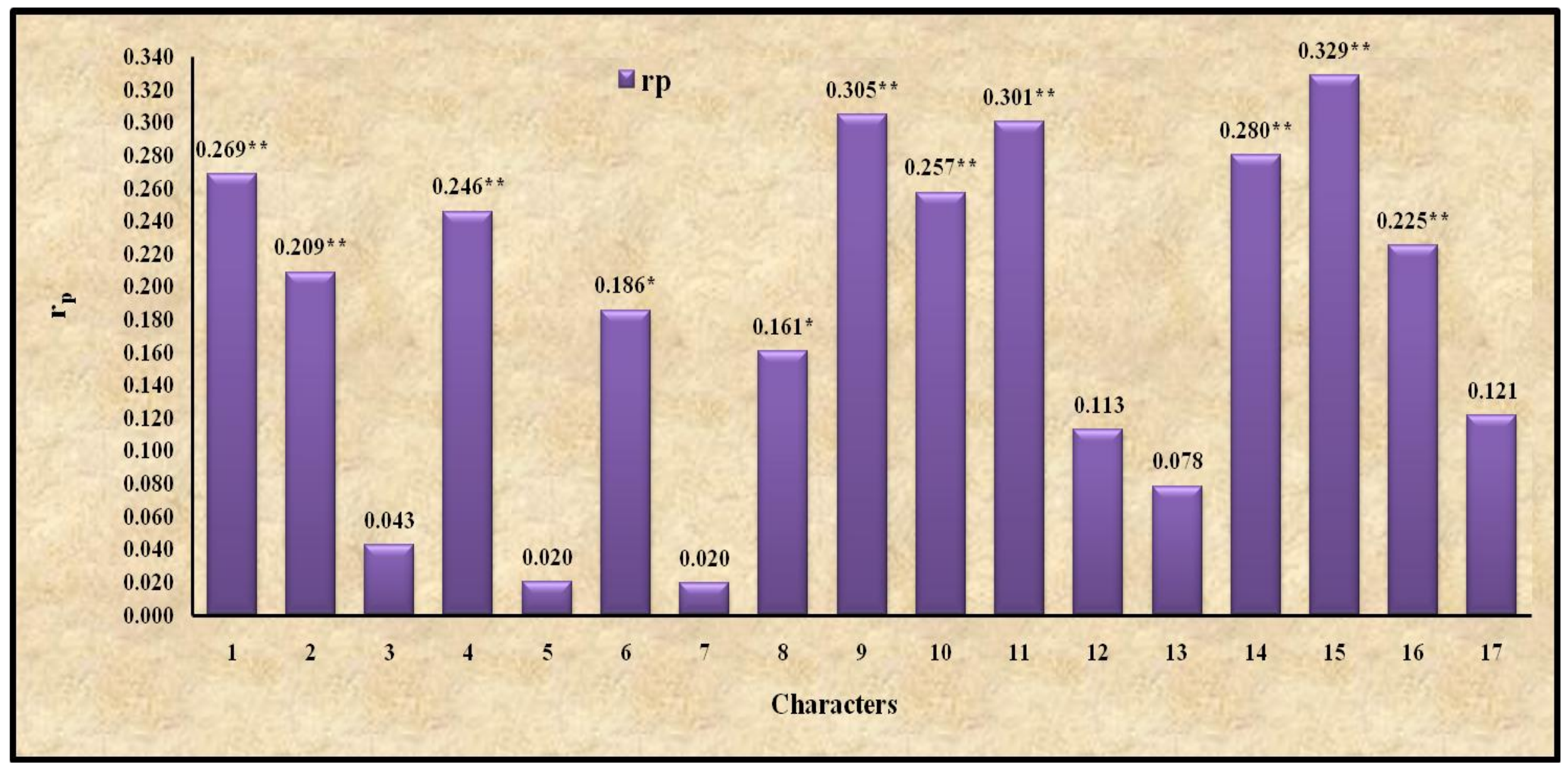

\begin{tabular}{|c|c|c|c|c|c|}
\hline 1. & Plant height (cm) & 2. & No. of leaves per plant & 3. & Leaf length (cm) \\
\hline 4. & Leaf width at middle portion $(\mathrm{cm})$ & 5. & Days to maturity & 6. & Pseudostem height (cm) \\
\hline 7. & Collar thickness $(\mathrm{cm})$ & 8. & Bulb collar diameter $(\mathrm{cm})$ & 9. & Bulb equatorial diameter $(\mathrm{cm})$ \\
\hline 10. & Bulb polar diameter $(\mathrm{cm})$ & 11. & Bulb weight (g) & 12. & No. of cloves per bulb \\
\hline 13. & Clove weight $(\mathrm{g})$ & 14. & Clove length $(\mathrm{cm})$ & 15. & Clove polar diameter $(\mathrm{cm})$ \\
\hline 16. & Clove equatorial diameter & 17. & Total soluble solids (\%) & & \\
\hline
\end{tabular}


The phenotypic path coefficient analysis revealed that plant height $(0.3108)$ followed by clove polar diameter (0.4153) exhibited high and positive direct effect on bulb yield and was found to be the most important yield components. The characters leaf width at middle portion (0.1489) and pseudostem height (0.1123) had low and positive direct effect on bulb yield, while bulb equatorial diameter (0.0468), bulb polar diameter (0.0334), bulb weight (0.0692), number of cloves per bulb (0.0056), clove equatorial diameter (0.0799), and total soluble solids (0.0975) had very low or negligible and positive direct effect on bulb yield. High and positive direct effect of plant height on bulb yield has also been reported in garlic by Srivastava et al., (1993), Barman et al., (1996), Agarwal (1999), Shaha and Kale (1999), Agrawal and Tiwari (2009), Meena (2010), Tsega et al., (2010), Singh et al., (2012), Dhall et al., (2013), Idress Al Gehani et al., (2013), Singh et al., (2013), Pervin et al., (2014) and Satesh Kumar et al., (2015), of clove polar diameter on bulb yield by Sharma et al.,(2016). Thus, these characters viz., plant height and clove polar diameter turned out to be the major components of bulb yield and direct selection for these traits will be rewarding for yield improvement.

\section{References}

Agrawal, M. K. (1999). Variability, correlation and path coefficient analysis in garlic. M.Sc. Thesis. Submitted to Rajasthan Agricultural University, Bikaner.

Agrawal, A. and Tiwari, R. S. (2009). Character association and path analysis in garlic (Allium sativum L.). Vegetable Sci., 36(1): 69-73.

Al-Jibouri, H. A., Miller, P. A. and Robinson, H. F. (1958). Genotypic and environmental variances in upland cotton cross of interspecific origin. Agron. J., 50: 633-635.
Baiday, A. C. and Tiwari, R. S. (1995). Character association and evaluation of garlic germplasm. Recent Horticulture. 2(2): 117-123.

Barman, D., Sharma, C. K. and Singh, I. P. (1996). Correlation and path analysis in garlic (Allium sativum L.). International Journal of Tropical Agriculture. 14: 247-250.

Bhatt, B., Soni, A. K., Jangid, K and Kumar S. (2017). A study on genetic variability and character association and path coefficient analysis in promising indigenous genotypes of garlic (Allium sativum L.). Int. J. Pure. App. Biosci. 5(1): 679-686

Dewey, O. R. and Lu, K. H. (1959). Correlation and path coefficient analysis of components of crested wheat grass seed production. Journal of Agronomy. 51:515-518.

Dhall, R. K. and Brar, R. S. (2013). Genetic vatiability, correlation and path coefficient studies in garlic (Allium sativum L.). Vegetable Sci., 40(1): 102104.

Esho Kamal Benyamin (2015). Performance of genetic parameters in garlic (Allium sativum L.). Res. J. Agr. Env. Sci., 2(2).

Federer, W.T. (1956). Augmented designs with one-way elimination of heterogeneity. Biometrics, 17(3): 447473

Idress Al Gehani and Adnan Kanbar (2013). Multivariate statistical analysis of bulb yield and morphological characters in garlic (Allium sativum L.). Australian Journal of Basic and Applied Sciences. 7(14): 353-358.

Kadam, S. S., Gadakh, S. S., Kathale, M. N and Kulkarni U. G. (2016). Correlation and path analysis for yield and yield contributing characters in garlic (Allium sativum L.). Society for Science and Nature. I.J.A.B.R. 6(2): 288-291.

Kalloo, G., Pandey, U. C., Lal, S. and 
Pandita, M. L. (1982). Correlation and path coefficient analysis studies in garlic. Haryana Journal Horticulture Science. 11: 97-102.

Khan, R. N. (2002). A study on genetic parameters in garlic. Horticultural Journal. 15(1): 75-80.

Kohli, U. K. and Fageria, M. S. (1992). Correlation studies of some important traits in garlic (Allium sativum L.) collections. Journal of Research APAU. 20(3-4): 164-168.

Kohli, U. K. and Mahajan Nutan. (1993). Yield performance and correlation studies in garlic a note. Haryana Journal of Horticulture Science. 22: 163-165.

Lokhande, C. D. and Pawar, B. B. (1988). Correlation studies in garlic. Journal of Maharashtra Agriculture University. 13: 110-111.

Meena, C. P. (2010). Genetic Variability, Correlation and Path Coefficient Analysis in garlic (Allium sativum L.). Thesis submitted to, Rajasthan Agricultural University, Bikaner.

Moravec, J., Kwasmicka, S. and Velicka, O. C. (1974). Correlation between bulb weight and other characters in varieties of the world collection of garlic. Bullentin Vyzkumy Utsav Zelinarsky, Oiomouc. 18: 15-23.

Panse, R., Jain, P. K., Gupta, A. and Sasode, D. S. (2013). Morphological variability and character association in diverse collection of garlic germplasm. African Journal of Agricultural Research. 8(23): 2861-2869.

Pervin, M., Kamrul Hassan Md; Hassan, K. and Hoque, AKMA. (2014). Genetic variation of indigenous, improved and exotic Allium sativum germplasm. Adv Plants Agric Res 1(2): 00011.

Prajapati, S., Tiwari, A., Jain, P. K., Mehta, A. K and Sharma, H. L. (2016). Evaluation and characterization of garlic (Allium sativum L.) genotypes. JNKVV, Jabalpur thesis.

Rahman, A. K. M. M. and Das, M. K. (1985). Correlation and path analysis in garlic. Bangladesh Journal of Agricultural Research. 10: 50-54.

Roy, D. (2000). Plant breeding - Analysis and exploitation of variation, Narosa Publishing House, New Delhi. pp. 135.

Samaptika, K., Sharma, P., Sarnaik, D.A., and Thawait, D. (2014). Studies on genetic variability and correlation coefficient of garlic (Allium sativum L.) genotypes under Chhattisgarh plain condition. National Academy of Agricultural Science.32: 1-2.

Satesh Kumar; Samnotra, R. K., Manoj Kumar and Khar, S. (2015). Character association and path analysis in garlic (Allium spp.) germplasm under subtropical environment of Jammu. An international Quarterly Journal of Life science. 10(4): 1997-2003.

Shaha, S. R. and Kale, P. N. (1999). Path coefficient analysis in garlic. Journal of Maharashtra Agriculture University. 24: 92.

Shaha, S. R., Kale, P. N., Dhankhar, B. S. and Shirsath, N. S. (1990). Variability and correlation studies in garlic. Haryana Journal of Horticultural Sciences. 19(34): 313-317.

Sharma, R.V., Komolafe, O., Malik, S., Mukesh Kumar; and Sirohi, A. (2016). Character association and path analysis in garlic (Allium sativum L.) International Quarterly Journal of Life science. 11(3):1931-1935.

Singh, R. K., Dubey, B. K. and Gupta, R. P. (2012). Studies on variability and genetic divergence in elite line of garlic, Journal of Spices and Aromatic crops 21(2): 136-144.

Singh, R. P. (1981). Genetic evaluation and path analysis in garlic. Madras Agriculture Journal of University. 24: 
92.

Singh, R. P. (1984). Association analysis in garlic. Journal of Research Assam Agriculture University. 65: 181-183.

Singh, S. R., Ahmed, N. A., Lal, S., Amin, A., Amin, M., Gaine, S. A. and Nusrat Jan (2013). Character association and path analysis in garlic (Allium sativum L.) for yield and its attributes. SAARC J. Agri., 11(1): 45-52.

Sonkiya, A. K., Singh, P. P. and Naruka, I. S. (2012). Variability, character association and path coefficient analysis in garlic, Medicinal plants, 4(2): 90-93.

Srivastava, B. P., Dubey, A. K. and
Srivastava, J. P. (1993). Genetic association and path analysis in garlic. Haryana Journal of Horticultural Sciences. 22(1): 85-88.

Thakur, J. C., Bhathal, G. S. and Gill, S. P. S. (1997). Genetic variability and correlation studies in garlic. Journal of Research Punjab Agricultural University. 34(1): 40-44.

Tsega, K., Tiwari, A., and Woldetsadik, K., (2010). Genetic variability, correlation and path coefficient among bulb yield and yield traits in Ethiopian garlic germplasm. Indian J. Hort. 67(4): 489499.

\section{How to cite this article:}

Pooja Chotaliya and Kulkarni, G.U. 2017. Character Association and Path Analysis for Quantitative Traits in Garlic (Allium sativum L.). Int.J.Curr.Microbiol.App.Sci. 6(8): 175-184. doi: https://doi.org/10.20546/ijcmas.2017.608.025 